\title{
Companions: Tests and Drug for Better Healthcare
}

\author{
Suman Mukherjee, PhD, and Sharon F. Terry, MA
}

$\mathbf{I}^{\mathrm{N}}$ N February 2014, the Institute of Medicine's Roundtable on Translating Genomic-Based Research for Health published a report on its workshop, "Refining Processes for the Co-Development of Genome-Based Therapeutics and Companion Diagnostic Tests." This workshop examined challenges and potential solutions for the codevelopment of targeted therapeutics and companion molecular tests for the prediction of drug response. With a desire to accelerate solutions, key stakeholders, including laboratory and medical professional societies, proposed solutions to resolve the concerns raised about codevelopment of companion diagnostic tests and therapies ahead of the meeting. Workshop speakers were given the proffered solutions before the meeting and were directed to comment on (1) whether the proposed solutions address the problems described, (2) whether there are other solutions to propose, and (3) what steps could be taken to effectively implement the proposed solutions.

Genomic data can be used to identify new drug targets for both common and rare diseases, can predict which patients are likely to respond to a specific treatment, and have the potential to significantly reduce the cost of clinical trials. Given the costs involved in drug development, strategies for creating efficiencies in the development processes are critical to the health of the industry, and, more important, in accelerating the delivery of interventions to the people who need them. Some authors have postulated that adoption of genomics-based approaches may in fact create some efficiencies.

Recently, the expectation of such benefits has led to the development and approval of several targeted therapeutics. A key component of each of these new drug approvals is the ability to identify the population who will benefit from treatment, which largely hinges on codevelopment. The codevelopment process has led to alterations in the way that some drugs are developed, resulting in close collaboration between pharmaceutical and diagnostic companies.

The Institute of Medicine's Roundtable on Translating Genomic-Based Research for Health workshop examined challenges and potential solutions for the codevelopment of targeted therapeutics and companion molecular tests for the prediction of drug response. The Roundtable provides a forum for diverse stakeholders to present approaches for assessing genomes and clinical use. The meeting compared and contrasted processes for evaluation evidence for different clinical indications and across stakeholders to identify key challenges and elicit pragmatic approaches to facilitate the effective translation of genomics into the clinic by improving evidence-based policy development.

The speakers addressed processes used to identify studies and data. They discussed selection criteria for tests/variants for full evidence review and assessment, how to assess data and synthesize it to form conclusions, how to present the results of evidence review and evaluation to policymakers, how to determine whether clinical action is recommended or taken for specific genomic variants, and how to define actionability. The speakers and audience debated the primary goal of determining the clinical utility and cost-benefit ratio of predictive genetic testing in order to ensure that this technology is used in an evidence-based fashion.

The workshop covered reimbursement, including how to evaluate genome or multigene panel sequencing. Discussion included examining criteria for coverage and the extent to which information reported in the electronic health record affects coverage decisions.

A section on guideline development evaluated nextgeneration sequencing. Speakers discussed how to use genetics and genomics to improve the care of patients through the promotion and implementation of personalized medicine.

A focus on patient care and decision-making considered patient preferences and understanding about what tests are done and what information is disclosed, and how well patients understand discussions about genomic testing. Investigators discussed pharmacogenomics in diverse populations, and decision modeling to inform research prioritization and stakeholder decision-making (including the evaluation of clinical, economic, and personal utility of whole genome sequencing). This led to a discussion of ideas and policy to initiate and educate healthcare providers, patients, and researchers.

Collectively, the goals identified by various speakers throughout the meeting included the following (National Research Council 2014):

- The development of a single test that could be used to simultaneously make a diagnosis, indicate treatment, and assess the adverse reaction risk for drugs upon clinical validation for each use.

- The creation of a global, value-based payment system for companion diagnostics (including next-generationbased testing) that would be based on evidence that

Genetic Alliance, Washington, District of Columbia. 
considers overall patient care and achieving specific outcomes.

- A learning healthcare system, that, with research and payer support, uses clinical data to improve patient care moving forward.

- The establishment of a national testing and outcomes database to generate evidence for improving clinical care.

- The implementation of regulatory guidance for nextgeneration sequencing that would allow for the development of tests and would both direct patient care and be used for drug trials.

- The development of a Food and Drug Administration process to alter the drug label to account for cleared or approved new tests for existing or new drugs that would provide an alternative to requests that are now driven only by pharmaceutical companies.

- The institution of a new reimbursement method that would account for next-generation-based testing to provide more patient data at the same or lower cost compared with multiple, individual diagnostic tests.

It is important that the solutions take advantage of the game change that genomics can initiate. As we have said over and over, these technologies, the stratification of populations that results, and the novelty of truly treating an individual can either cause greater cost burden and disparities or offer a new avenue to efficiencies and equities. If the same stakeholders look for the same return in the same manner as they have in the past, then we will not see the promise realized. It is time for every stakeholder-pharmaceutical companies, test developers, regulators, payers, and patients - to consider an integrated system in which all have a role, each contributes learnings to the system, and each risks new ways of working together. It is time to stop protecting our turf and join in creating the world we hoped for, in designing novel solutions that really help to heal our broken healthcare system.

\section{Reference}

National Research Council (2014) Refining Processes for the Co-Development of Genome-Based Therapeutics and Companion Diagnostic Tests: Workshop Summary. Washington, DC: The National Academies Press, p. 51.

Address correspondence to: Sharon F. Terry, $M A$

President \& $C E O$

Genetic Alliance

4301 Connecticut Avenue, NW

Suite 404

Washington, DC 20008

E-mail: sterry@geneticalliance.org 